\title{
LAS ANCIANAS EN ESTADOS UNIDOS: MITOS Y REALIDADES*
}

\author{
Elizabech W. Markson \\ Universidad de Boston
}

\section{INTRODUCCION}

La población de los Estados Unidos, al igual que la potlación del resto del mundo, está envejeciendo rápidamente. Veintinueve millc nes de americanos, más de uno de cada diez, tienen ahora sesenta y cinco años o más. En el 2030, uno de cada cinco americanos se encontrará en esta categoría de edad. La explosión de la población entre las mayores se hace especialmente evidente entre las mujeres de una muy avanzada edad que sobreviven hasta los ochenta y cinco años o más en unos números y proporciones sin precedientes. El dramático aumento, tanto en los EE.UU. como en otros lugares, de mujeres que se haIlan entre las edades de sesenta y cinco y ochenta y cinco a ios plantea problemas críticos a los gerontólogos, a los políricos y, en general, a todos nosotros. Sus situaciones pueden ofrecernos información sobre la ac ual calidad de vida de los ancianos, al igual que sobre la política que afecta a.l envejecimiento y la familia en el futuro. Este artículo revisa los mitos que af actan a las ancianas americanas al igual que su estado civil, la composición familiar, su plan de vida, renta y empleo, ayudas familiares, ciudados otorgades y recibidos. Aunque Alemania Occidental y Francia poseen en la actualidac unas proporciones mayores de ancianos, los EE.UU. las sobrepasarán en las próximas décadas (1). Se espera que en el año 2030, el número de personas de más de ochenta y cinco años en los EE.UU. casi cuadruplique su número, tendencia que se proyecta continuará a lo largo de todo el siglo veintiuno. Cada vez hay más americanos que llegan a los noventa años y los sobrepasan: en 1986, según las apreciaciones oficiales del censo, eran centenarias unas veinticinco mil personas. Dados los actuales modelos de esperanza de vida, la población anciana

* Reproducimos, por su interés, este articulo ya publicado en el rúmero l del año 1991 de la Revista de Gerontologia, a quienes agradecemos la autorización pas a poder editarlo en este volumen de PAPERS. 
"Papers»: Revista de Sociologia

creciente de los EE.UU. se hallará compuesta principalmente por mujeres. Las mujeres americanas de más de sesenta y cinco años superan en la actualidad en una proporción de tres a dos a sus iguales masculinos, un cambio considerable respecto a hace escasamente treinta años, cuando la proporción de mujeres en relación a la de hombres era de seis a cinco. Entre los muy ancianos (85+) hay cinco mujeres por cada dos hombres. Claramente, la tercera edad es un territorio habitado por las mujeres durante mayores periodos de tiempo.

\section{MITOS QUE AFECTAN A LAS ANCLANAS}

\section{La variable proporción de dependencia y el mito de la avaricia}

Uno de los principales desafíos que presenta la mayor longevidad, tanto de hombres como de mujeres, es el de que América es una nación donde se valora la vida larga pero donde la vejez no es bien recibida. Dado que la mortalidad durante la niñez y la infancia se ha reducido drásticamente, la vejez ha empezado a asociarse cada vez más con la enfermedad y la muerte, algo temido en una sociedad tan individualista, consciente de su juventud y orientada hacia la actividad como es la sociedad de los EE.UU. Además, en las sociedades postindustriales normalmente no se necesita el trabajo de los mayores y su sabiduría ha sido sustituida por la educación formal y las tecnologías de la comunicación. A finales de los setenta, los economistas conservadores empezaron a señalar la creciente "carga" de tener que mantener a una población de ancianos (2). Durante un periodo de restricción fiscal en los servicios sociales y humanos, la llamada "equidad generacional», descrita por uno de sus principales defensores como unada más que el presente sea justo con el futuron(3), se ha convertido en el tema de moda. Durante los ochenta, la tercera edad se convirtió en la cabeza de turco económica $(4,5)$, descrita como avariciosos que se enriquecían a expensas de los niños, y que recibían una cantidad desproporcionada de los beneficios de las inversiones federales. Como afirmó un anterior gobernador de Estado, Richard Lamm (principal portavoz del recientemente formado grupo lobby, Americans for Generational Equtity): "Debemos reconocer que existe una relación entre los presupuestos para los ancianos y los presupucstos para los jóvenes... El dineto que necesitan urgentemente los niños pobres de St. Paul se envía a los ricos jubilados de St. Petersburgon (6).

En efecto, dado que los americanos no sólo viven más sino que también tienen menos hijos, la composición de la "proporción de dependencia", es decir, el número de gente por debajo de la edad de dieciocho años y por encima de la edad de sesenta y cuatro con respecto al de las edades activas entre los 
dieciocho y sesenta y cuatro, ha cambiado notablemente durante el siglo veinte. El promedio de hijos por familia americana en el año 1900 era de cuatro; hoy el promedio se encuentra ligeramente por debajo de los dos. En 1900 había siete ancianos por cada cien personas en edad activa; ahora hay unos veinte ancianos por cada cien en edad activa. Por primera vez en la historia, el matrimonio medio en los Estados Unidos tiene más padres que hijos (7). Se espera que esta tendencia aumente, con cada vez menor núnero de personas en edad activa que mantengan a los mayores. Olvidada por defensores de la equidad generacional preocupados por las contenciones de costes, se halla la cuestión de que el atımento de ancianos que deberán ser mantenidos se halla contrarrestado por una disminución casi equivalente en la p oporción de niños a mantener, debido a unas tasas de natalidad más bajas. Cilaramente, el coste económico de los ancianos dependientes se halla equilibrało por los costes reducidos de una menor población dependiente infantil.

Irónicamente, aunque se ha considerado a los ancianos como consumidores avariciosos de los recursos, no se ha promulgado ningu ua legisiación en los Estados Unidos desde 1972 que les beneficie. Las retribuciones de la Seguridad Social - la mayor fuente de ingresos para las an:ianas- se ha vuelto imponible y los incrementos del coste de la vida meno: generosos ${ }^{1}$. Desde sus comienzos, "Medicare" --un programa de fondos ferlerales que proporciona cuidados médicos a todos aquellos de más de sesent a y cinco años- ha aumentado de forma continuada la cantidad de deducibles, copagos y primas que deben pagar los partictulares de su propio bolsillo. El recientemente revocado Decreto Medicare sobre el Alcance de la Salud Catastrófica de 1988, por ejemplo, tuvo como premisa fiscal subyacente que los ancianos correrían totalmente con el gasto de los nuevos beneficios mediante i.xcrementos uniformes en el pago de la aseguranza médica suplementaria mensual más de las primas anuales, que eran imputstos adicionales en la carga de impuestos sobre la base imponible de los ancianos. Se ha propuesto la racionalización de la asistencia sanitaria a los ancianos para contener los costes (política que ya se ha llevado a cabo en algunos otros países: la diálisis de riñón y los trasplantes de corazón en Gran Bretaña).

Como ha expresado muy duramente Richard Lamm, "Los ancianos tienen la obligación de moritse y quitarse de en medion una afirmación moderada más tarde con un "Simplemente no tratamos con mucha madurez el tema de la muerte y de morir... y una fuente de dinero reasignable son los dólares que

1. La Seguridad Social en los Estados Unidos no está financiada port los ingresos generales, sino que se trata más bien de un «sistema de retención fiscal en la fuentrm en el que los jubilados de hoy son mantenidos por los impuestos sobre la base imponible a lis trabajadores actuales, al igual que las contribuciones de los jubilados se usaban para financiar los beneficios de anteziores grupos de trabajadores jubilados. 
se están gastando con los enfermos terminales... Para mi no tiene sentido hacer trasplantes de corazón a ex-fumadores de sesenta y cinco años" (6).

La racionalización del cuidado sanitario para los ancianos ha sido explicada en mayor profundidad por el moralista Daniel Callahan (8), que propuso la negación de los cuidados sanitarios que alarguen la vida de todos aquellos entrados en los setenta o más. La racionalización de los recursos de asistencia sanitaria, económicos y sociales para cualquier segmento de la población tiene profundas implicaciones morales y sociales, no sólo para aquellos a los que les son negados sus beneficios, sino para toda la sociedad en su conjunto. Con mayor inmediatez son las ancianas y sus hijos de mediana edad o a principios de la edad madura los más afectados por la premisa de que los ancianos son egoístas, no dispuestos a llevar su propia carga, reduciendo los recursos sociales que pertenecen con razón a los jóvenes.

\section{La edad de oro perdida y el abandono familiar}

Además del nuevo mito de la avaricia de los ancianos, al menos otros dos mitos sobre los ancianos y sus familias son corrientes en los Estados Unidos, y ambos esconden las condiciones reales de las ancianas. En primer lugar, existe el mito de la "edad de oro" perdida cuando los ancianos eran auromáticamente amados y respetados (10). Más bien, en aquellas sociedades donde el status de los ancianos había sido alto, su poder derivaba precisamente de su control sobre los bienes y los recursos (1 I): situación no disfrutada a menudo por los miembros más jóvenes de la familia.

En segundo lugar, existe el mito del abandono familiar de los ancianos. Aunque el tipo familia-clan nunca ha sido la forma de familia dominante en los Estados Unidos (12), se han acumulado numerosos indicios que indican que los miembros de la familia continúan siendo la principal fuente de ayuda entre ellos durante el curso de sus vidas, con el movimiento de recursos de los más capacitados a los menos (13-16). Aunque los recientes descensos en la fertilidad han tenido como resultado unas familias más pequeñas, de manera que los ancianos pueden confrar menos en la ayuda de los miembros de la famitia, diversos estudios han mostrado la persistencia de los lazos familiares, demostrada por los altos índices de correspondencia, Ilamadas telefónicas y visitas, y por las expresiones de interés por el bienestar de sus padres por parte de los hijos $(17,18)$. En la actualidad un $80 \%$ de los americanos de mediana edad tienen al menos un padre vivo comparado con menos de la mitad en 1900. También es cada vez más probable que incluso aquellos con sesenta y cinco o más años tengan al menos un padre que les sobrevive, y las familias de cuatro y cinco generaciones se están volviendo cada vez más normales. A pesar de la 
tendencia a una mayor movilidad residencial, especialmente entre los hijos adultos de las familias de clase media alta y aquéllos con posibilidades de ascenso social, unos ocho de cada diez mayores con hijos viven a una hora de distancia en coche de al menos un hijo. La red de ayuda social a la mayoría de los ancianos consta así de un centro de miembros familiıres con los amigos $y$ los vecinos en la periferia (19).

Los miembros ancianos de la familia también proporcionan apoyo emocional, y a veces económico, a los hijos adultos solteros, dirorciados y viudos, que es más probable que tengan contactos cara a cara frecuentemente con un padre anciano que con sus hermanos casados (20). Típicantente, sin embargo, los ancianos prefieren un modelo de "intimidad a distancia" con hijos (21); es decir, mantener su independencia viviendo cerca pero no con sus hijos. En efecto, datos longitudinales muestran que las personas mayores consideran que vivir solas es ahora más deseable que hace tres décadas y, para mantener su independencia, están más predispuestas a sacrificar los ingresos que obtendrían al trasladarse a vivir con otros (22).

\section{La mayoria de los ancianos están en instituciones}

Unido al mito del abandono familiar está la creencia de que una alta proporción de ancianos vive en asilos u otras modalidades de asistencia a largo plazo. Lo contrario es cierto; sólo un $5 \%$ de ancianos se e..cuentra en alguna de estas modalidades de asistencia a largo plazo en algún momento de su vida. Más o menos dos tercios de los ancianos viven en un amb entc familiar. Pero existen marcadas diferencias de sexo. Los hombres tienden a vivir con sus esposas hasta que mueren, pero sólo poco más de un tercio de mujeres mayores de sesenta y cinco años viven con stus maridos. De hecho, más o menos tres cuartos del $5 \%$ de ancianos que han estado alguna vez en su vida en asilos son mujeres. El residente típico de un asilo es una mujer blanca, viuda de ochenta y cinco años o más, con múltiples incapacidades, admitida procedente de una corta estancia en el hospital y más propensa a haber vivido sola antes de su admisión. Por el contrario, sólo un tercio de los hombres en asilos eran viudos en el momento de su admisión, y un $21 \%$ nunca habían estado casados (23). Es menos probable que las ancianas afroamericanas y latinizs ingresen en asilos u otras modalidades de asistencia; en consecuencia, sus farnilias soportan mayores cargas por sus cuidados cuando quedan seriamente incapacitadas (24). Aquéllas de cualquiera de estas razas o sexo que son admi idas en un asilo, cs más probable que tengan hijos vivos que los ancianos dentro de ta comunidad, destacando el papel crítico del apoyo familiar en preveni.: o retrasar la institucionalización. 
"Papers": Revista de Sociologia

\section{SITUACIÓN DE VIDA DE LAS ANCIANAS}

\section{Estado civil, composición familiar y plan de vida}

Estar casado tiene diferentes consecuencias para los hombres y las mujeres durante el curso de sus vidas, y, en la tercera edad, estar casado parece más crucial para la interacción social, la esperanza de vida y la ausencia de síntomas depresivos entre los hombres que entre las mujeres $(25,26)$. Para ambos sexos en la tercera edad, la ausencia de hijos en el hogar se halla asociada a tna mayor compenetración y compañía entre marido y mujer (25).

El plan de vida de las mujeres àncianas varía según los grupos raciales y étnicos. Hay una mayor tendencia entre las ancianas afroamericanas y latinas a vivir en el centro de la ciudad que entre sus homólogas de raza blanca. Las ancianas latinas que viven en el noreste están concentradas cn áreas urbanas pobrcs de ciudades tales como Nueva York, Filadelfia o Boston, y comparten el español como su mayor lazo común; sus antecendentes de inmigración varían tan ampliamente como sus países de origen (27). Las ancianas asiáticas y de las islas del Pacífico constituyen un grupo muy diverso, incluyendo chinas, japonesas, coreanas, vietnamitas, laosianas, filipinas y de Hmong entre orras; como las latinas, las últimas inmigrantes de Asia y del Pacífico se encuentran más a menudo concentradas en la ciudad, en vecindarios de bajos ingresos. A pesar de sus diferencias en el idioma y país de origen, las inmigrantes de Asia y de las islas del Pacífico comparten los temas comunes de los papeles estrictamente ligados a determinada edad y sexo, menor autoridad de las mujeres y la prioridad de las necesidades de la unidad familiar sobre las del miembro individual (28). Las viudas afroamericanas, de Asia o de las islas del Pacífico, tanto urbanas como rurales, tienden a vivir con otros miembros de la familia, mientras que las viudas de raza blanca a mantener familias separadas (29). Estas diferencias reflejan tanto la tradición cultural como las condiciones socio-económicas. Las mujeres no latinas de raza blanca tienen una mayor tendencia a vivir con hijos cuando están incapacitadas, pero un minoritario grupo de ancianas, incluyendo un número desproporcionado de las muy pobres, están más predispuestas a vivir con miembros de la familia debido a sus desventajas económicas $(24,30)$.

De los 8,5 millones de ancianos ameticanos que viven solos, las mujeres comprenden casi cuatro quintas partes. La mayoría son viudas, teniendo en cuenta que tres de cada cuatro mujeres entre los sesenta y cinco y setenta y cuatro años viven solas, y más de cuatro de cada cinco tienen más de setenta y cinco años (31). Muchas de las ancianas que viven solas no tienen hijos vivos, una situación más común entre las afroamericanas que entre las de raza blanca. Sin tener en cuenta el estado civil, la mayoría de las mujeres entran en la vejez con un gran apoyo social acumulado a través del intercambio social 
a lo largo de sus vidas (32). Las solteras, contrariamente a a creencia popular, no están aisladas socialmente y no por eso tienen tamporo menor actividad social que las casadas, viudas o divorciadas $(33,34)$. Las ancianas solteras manifiestan un más alto nivel de interacción con sus amigos que las casadas, y son las más propensas de cualquier grupo marital a tener hermanos o hermanas u otros parientes en casa (34). Paradójicamente, el ingreso económico no es el factor principal en los contactos sociales, tanto con lo:i amigos como con la familia; mientras que los contactos sociales están directamente relacionados con un mayor ingreso económico entre las ancianas de raza blanca, la proporción es inversa entre las afroamericanas (35).

\section{Participacion en la fuerza de trabajo e ingresos}

Aunque el reingreso al trabajo de las mujeres de mediana edad y de las más mayores se ha incrementado durante los últimos años, sólo un $20 \%$ de las mujeres entre los cincuenta y sesenta y cuatro años habia trab:ijado con continuidad (veintiséis semanas o más por año desde 1987 a 198:), el 13\% no tenía experiencia laboral alguna, y los restantes dos tercios tuvis:ron varios tipos de trabajos (36). El número de americanas trabajando por un salario mínimo (que no ha variado en la pasada década) se incrementó er. 2,7 millones entre 1981 y 1987. Las mujeres continúan concentradas en las ouupaciones peor pagadas; seis de cada diez de aquéllas con cincuenta y cinco años o más y la mitad de aquéllas entre los veinte y treinta años trabajan en ventas al por menor, tareas administrativas y clericales y servicios, áreas todas ellas que pagan salarios por debajo de la media nacional para todos los emploos (37).

Actualmente las mujeres entre los cuarenta y sesenta y cinco años están en particular desventaja en la situación laboral, ya que han experimentado más abiertamente la discriminación sexual, menos oportunidad ss para la formación en el trabajo y la discriminación por la edad (38). Las implicaciones de la posición relativamente en desventaja de las mujeres de mediana edad en el terreno laboral es, pues, que los hombres continúan siendo capace.; de mantener o encontrar empleo en industrias siendo, además, mejor pagado; y teniendo mejores beneficios en la jubilación que las mujeres. EI Decreto pará la Reforma de Impuestos, aprobado en 1986, facilita créditos de impuestos en una escala móvil a personas con ingresos muy bajos, pero elimina la exención extra personal anteriormente facilitada a todos los mayores de sesenta y cinco años: otro inconvenientc para aquellas mujere; que no sufren extrema pobriza pero tienen sueldos limirados o jubilaciones anticipadas limitadas. Tampoco se espera que mejore la situación financiera para las mujeres mayores en ..os próximos treinta años. Cada vez, más, la pobreza en los Estados Unidos se ha feminizado, con ma- 
dres solteras con hijos jóvenes y mujeres ancianas entre las más pobres de la nación. De acuerdo con las predicciones actuales, las personas que serán pobres en el año 2020 serán principalmente mujeres ancianas que dependerán de la Seguridad Social y dell Ingreso de Seguridad Suplementario (un programa de manutención por ingresos probados para ancianos e imposibilitados, pagando individual mente un máximo aproximado de 4.842 dólares por año (en dólares de 1988), un $25 \%$ por debajo del nivel de pobreza de 5.649 dólares por persona) ${ }^{2}$.

Comparado con los ancianos, las ancianas de cada uno de los estados civiles tienen ingresos económicos más bajos, ampliamente asociado al modelo de dependencia económica de por vida de los hombres y a la viudez en la tercera edad. En 1987, por ejemplo, las mujeres ancianas comprendían el $72 \%$ de los ancianos pobres y tenían menos posibilidades de recibir el Ingreso de Seguridad Suplementario. El ingreso medio en dólares en $1987-6.425-$ para todas las ancianas, era sólo el $56 \%$ del de los hombres (I 1.544 dólares). Las más vulnerables a la pobreza son las viudas ancianas, especialmente las muy ancianas y las que son miembros de minorías. Más de la mitad de las ancianas afroamericanas y latinas que no viven con miembros de la familia, rienen ingresos por debajo del nivel de pobreza; la mitad de todas las mujeres de setenta y cinco años o más que viven solas, subsisten con ingresos un $150 \%$ por debajo del nivel de pobreza.

Paradójicamente, a pesar de las acusaciones de los defensores de la equidad generacional, los ancianos de los Estados Unidos obtienen una proporción menos pequeña de sus ingresos a los programas de seguros sociales del Gobierno, tales como la Seguridad Social, que los ancianos de otros países desarrollados (1). Las tasas de pobreza de los ancianos en los Estados Unidos en general, y de las ancianas en particular, se encuentran entre las más altas en el mundo desarrollado (1). Cuando se ajustaron los ingresos a una base equivalente a sicte países, sólo Israel tuvo una mayor concentración de bajos ingresos en los ancianos que Esrados Unidos.

\section{Cuidadores y cuidados en la familia}

Son principalmente las mujeres las que dan y reciben asistencia en la tercera edad. Aproximadamente el $72 \%$ de las asistentas son mujeres, el $29 \%$ de

2. Al interprezar los niveles de pobreza, es importante tener en cuenta que el gobierno federal aplica diferentes ripos de renta para los menores y los mayores de sesenta y cinco años. Se considera que los mayores necesitan menos dinero; en 1987 el indice de pobreza de una pareja anciana se calculaba en ochocientos dólares menos en la renta anual que la de una pareja menor de sesenta y cinco años. Para individuos de sesenta y cinco años o más, el índice era de cuatrocientos ochenta y un dollates menos por año. 
las cuales son hijas y el $23 \%$ esposas (29). La mayoría de la asistencia a largo término basada en la comunidad, la proveen miembros de la familia, con sólo un $5 \%$ de cuidados recibidos de fuentes pagadas.

Entre las parejas ancianas la cuidadora es, con doble orobabilidad, la esposa, seguida de las hijas, otros parientes y otras fuentes ce asistencia pagada $(17,39)$. Dichos cuidados entre las parejas casadas son más a menudo llevados a cabo por la esposa, lo que refleja en parte la mayor esperanza de vida de las mujeres; esto también refleja la persistencia de los roles sexuales rradicionales entre los miembros de la familia. Las mujeres tienden más : realizar tareas tales como bañar, vestir, limpiar, cocinar y los trabajos de la casa. Los asistentes masculinos, a menudo ofrecen servicios típicamente "masiulinos" tales como el transporte, reparaciones en el hogar, administración f nanciera y soporte económico (40,41). Los ancianos seriamente incapacitados que viven con sus parejas reciben servicios pagados sólo cuando el otro miembro de la pareja está incapacitado mental of físicamente (42).

Sin embargo, la mayoría de los ancianos inhabilitados en la comunidad, son mujeres, muchas de las cuales enviudan después de haber atındido a su cónyuge enfermo. De acuerdo con los datos de la investigación realizada por el National Nursing Home, una mayor proporción de ancianas que de ancianos admiten dificultades en las actividades de asistencia personal tales conno el baño, el vestir, etc. Estas dificultades se hacen más patentes al incrementar ia edad, aunque más de la mitad de las mujeres de ochenta y cinco años o más indican que realizan todas las actividades de asistencia personal sin dificultad. L as mujeres también admiten una mayor dificultad al realizar tareas de administr.ción del hogar, quizás reflejando en parte el hecho de que la mayoría de las ancianas no realizan tareas de este tipo o, si lo hacen, dudan más en admitir incapacidad en las encuestas de investigación. Esas ancianas tienen un status funcional peor que el de los hombres; cuando la edad se mantiene constante puede también teflejar la asociación de por vida entre la pobreza femenina y la salud débil durante el curso de la vida. Los ancianos pobres son doblemente propensos a tener limitaciones relacionadas con la salud en tareas de asistencia personal o domésticas (31).

Cuando una esposa no está disponible o no puede cuidar de un padre anciano, los miembros familiares activan una jerarquía de se ección de cuidadores, con una hija (o nuera si no hay hija) en cabeza, seguida de otros miembros familiares, quizás vecinos y amigos y, finalmente, recursos exteriores $(43,19)$.

Los cuidados no son, por lo general, repartidos equitativamente entre los miembros de la familia $(44,45,18)$. La proximidad a un anciano necesitado de ayuda, menos trabajo competitivo y obligaciones familiares, sentimientos de agradecimiento hacia los padres y normas culturales y familiares también juegan su papel $(46,29)$.

De los cuidadores que viven en casa, poco más de seis dì cada diez son hijos 
"Papers": Revista de Sociologia

e hijas, aproximadamente la mitad de los cuales indica que no vivirian en casa si su ayuda no fuera necesaria. Un $10 \%$ adicional de asistentes informales que no viven con la persona enferma se traslada para estar más cerca y ofrecer su ayuda.

Para las mujeres ancianas que viven solas, los parientes son su principal fuente de ayuda. Aproximadamente dos tercios sienten que pueden depender de la ayuda de sus familiares - a menudo sus hijos-durante unos días, y la mayoría puede contar con ayuda familiar durante unas semanas (31).

No importa si el cuidado es esposa, hija u otro familiar, una creciente literatura ha documentado los efectos adversos de proporcionar cuidados (47, 51). Las hijas al cuidado de sus padres ancianos experimentan niveles más altos de estrés que los hijos (52); y es más probable que las hermanas de los principales cuidadores de ancianos se sientan más estresadas que los hermanos ( 53 , 54). La mayor presión denunciada por las mujeres puede derivar de los lazos emocionales más intimos entre madre e hija $(55,56)$, por el hecho de que los hombres son menos dados a compartir sus sentimientos (54), o por su menor intimo contacto físico con tareas de cuidado diario, como bañar, vestir y asear. Es probable que las asistentas femeninas valoren su salud física peor de lo que lo hacen las mujeres que no dan tales cuidados; según un estudio, un tercio de las mujeres cuidadoras entre cuarenta y cinco y sesenta y cuarro años evaluó su salud en conjunto como normal o débil comparada con poco más de un quinto de no cuidadoras femeninas de la misma edad (29).

Los que reciben atención también sufren estrés. Los ancianos que viven en hogares con hijos adultos o nietos presentan una mayor tendencia a tener una moral y satisfacción más baja que los ancianos viviendo en otro entorno. Aunque sus niveles más altos de insatisfacción están sin duda influidos por sus enfermedades, la pérdida de independencia entre los ancianos que siempre han tenido un modelo de vida independiente no debería ser minimizada (25).

Las últimas recomendaciones de la Pepper Commission en el Congreso, proponian una doble aproximación a la asistencia a largo plazo, que mitigaría parte del estrés asociado normalmente a cuidar y ser cuidado dentro de la familia, especialmente entre los muy pobres. Concentrado en los cuidados sanitarios en casa y asilos, el informe de la Comisión propuso que la asistencia en el hogar estuviese a disposición de los gravemente incapacitados sin zener en cuenta la edad, estando esos beneficiarios capacitados para pagar un $20 \%$ de los costes y los subsidios estatales a disposición de aquéllos con ingresos inferiores a dos veces el nivel de pobreza. Similarmente, la asistencia en asilos sería suministrada mientras fuese necesitada al pagar los beneficiarios el $20 \%$ de los costos durante los tres primeros meses; de nuevo, los subsidios serían destinados a aquéllos cuyos ingresos fueran más bajos que el doble del nivel de pobreza. Pasados tres meses, el Gobierno pagaría todos los gastos de asilo de aquéllos con pensiones por debajo de los 30.000 dólares para un solo in- 
dividuo y de los 60.000 dólares para parejas (excluyendc el valor en dólares del hogar). Los déficits en el presupuesto federal más las reducciones en el presupuesto para el servicio humano hacen improbable la ajrobación de tal legislación en un futuro inmediato.

\section{DISCUSION}

En la tercera edad, las mujeres americanas poseen fuertes lazos familiares a los que pueden acudir en caso de necesidad. Pero también son vulnerables: más propensas a ser pobres, a enviudar, a tener limitaciones funcionales y, al final de su vida, a pasar sus últimos días en un asilo. Parece improbable que las políticas verdaderamente favorables a las ancianas apzirezcan hasta que el actual consenso ideológico no haya dado paso a una atención más amplia a la vulnerabilidad de las mujeres en todos los grupos.

¿Qué hay de la equidad generacional? Un libro reciente creado por dos defensores de este concepro argumenta: "Somos la primera sociedad en la historia que socializa los gastos de la tercera edad mientras que deja los gastos de los niños en crecimiento a entidades en su mayoría privac as... Como nación nos sentimos enormemente orgullosos por sacar a ambos padres del hogar $\mathrm{e}$ introducirlos en el terreno laboral; por tranquilizar a los ́ncianos estadounidenses de que nunca tendrán que depender de sus hijos; por limpiar el concepto de la jubilación universal y anticipada de toda conrotación negativa, y por alquilar un ejército de los profesionales mejor pagades en el mundo para ser pioneros en los trasplantes de órganos en la era espacial mientras se permite que un millón de mujeres embarazadas se conformen tan sólo con una visita médica al año. Llegado este punto, debemos pararnos y preguntarnos a nosotros mismos: ¿Es esta dirección sostenible?n (55).

La cuestión de si la actual política social americana sobre asistencia sanitaría a mujeres embarazadas es justa, es cierramente válid:a. La tasa de mortalidad infantil en los Estados Unidos es de alrededor del 9,9 por 1.000 nacimientos: una tasa de mortalidad dos veces mayor que la del Japón y casi dos veces mayor que la de Suecia (56). Que una clase de gente particularmente vulnerable -en este caso los ancianos-- sea considerada responsable del fracaso en la provisión de asistencia para otras categorías soviales tales como las mujeres embarazadas es, sin embargo, un giro interesante en culpar a un grupo de víctimas por la victimización de otro grupo. Generalmente, poco se hace para transferir ingresos a los más necesitados de cualquie:: edad, y los más ri$\cos$, un quinto de la población estadounidense, continúan teniendo más de nueve veces el ingreso del quinto más pobre. Cada vez más, las familias con hijos jóvenes se componen de parejas con dos sueldos; el 57\% de las mujeres 
"1'apers": Revista de Sociologia

casadas con niños menores de seis años y casi el $73 \%$ de las mujeres casadas con hijos entre los seis y diecisiete ańos se encontraban en situación laboral en 1988 comparado con el 30\% y $42 \%$ en 1970 (57). Durante la década de los ochenta, los subsidios federales y estatales para la asistencia al niño fueron dramáticamente reducidos, limitando el acceso a la asistencia diaria a las familias pobres y casi pobres especialmente aquéllas con madres solteras como cabeza de familia (58).

Las recientes reformas del impuesto sobre la base imponible han asegurado una mayor riqueza para aquellos que menos lo necesitan; las reducciones de impuestos de base de oferta de 1981 destinadas a potenciar la productividad, significaron que muchas empresas pagaran poco o no pagaran el impuesto federal sobre la base imponible. En 1983, la mitad de un uno por ciento de las familias estadounidenses pagaron un $35 \%$ del valor neto total de las familias americanas (59), y el 10\% de los poseedores de riqueza tenían en su poder dos tercios del capital privado $(60,61)$. Tampoco las reformas del impuesto sobre la base imponible de 1986 cambiaron sustancialmente la acumulación de riqueza entre los muy ticos. Entre 1978 y 1987 los ingresos del $20 \%$ más bajo del total de las familias americanas descendió casi un $10 \%$, y el $20 \%$ de las más altas se incrementó en más de un 15\% (62); los beneficios de los servicios sociales para familias pobres con hijos pequeños fueron reducidos e incomprensiblemente ajustados. En comparación con otras naciones desarrolladas, los Estados Unidos de América gastan proporcionalmente menos del presupuesto anual del Estado en programas de servicios sociales para personas de todas las edades, y cada vez más están dirigidos a apoyar programas militares y a beneficiar la deuda nacional que a programas de asistencia y ayudas sociales (63). Los dos programas más importantes que favorecen a la tercera edad - Seguridad Social y Medicare - son en gran medida autofinanciados; es decir, que quienes hicieron las contribuciones a estos dos fondos fueron los consiguientes beneficiarios de tales fondos y esto representa impuestos retrasados más que beneficios del presupuesto general.

Los debates políticos designados a crear falsas dicotomías, como los derechos de los nonatos y niños de corta edad respecto a la tercera edad, oscurecen las realidades actuales de la desigualdad económica. Las mujeres, que en todas las edades tienden a ser más pobres que los hombres, son puestas en contra de sus propios intereses. Si realmente nos preocupa la tercera edad, la familia y mujeres de todas las edades, las consecuencias de la feminización de la pobreza y la desigualdad estructural deben ser atendidas. La discriminación por edad o raza, la igualdad salarial para las mujeres, la formación y reeducación laboral para ambos sexos, el reparto desigual de la riqueza, una adecuada pensión durante el curso de la vida, incluyendo la tercera edad, y un sistema de asistencia sanitaria universal para personas de todas las edades; éstos son pun- 
tos que afectan no sólo a la calidad de vida de las ancianas, sino también a las oportunidades de vida de todos. Hay una aguda, a menuco olvidada pero vital, diferencia entre la política basada en los prejuicios centra los ancianos y la política que beneficiaría la calidad de vida de grupos de cualquier edad. Menospreciar los derechos de las mujeres ancianas es abrogar sus contribuciones pasadas al presente y al futuro que ellas han construido. jeguramente, ellas, sus hijos y nietos, que liegarán algún día a la vejez, se rerecen ajgo mejor.

\section{BIBLIOGRAFIA}

1. Torrey, B.B.; Kinsella, K.G. y Taueber, C.M. (1988), An a.ring world, Washington DC, US Bureau of the Census.

2. Samuelson, R.J. (1978), "Aying America: Who will shoulder the burden?", National Journal, 10, pp. 1712-1717.

3. Durenberger, D. (1989), "Education and the contract berween the generations", The Generations Joumal, 11 (1), pp. 5-8.

4. Binstock, R.H. (1983), "The aged as scapegoat", Gerontologisi, 23 (2), pp. 136-141.

5. Binstock, R.H. (1988), "Aging, politics and public policy", The world and I, 3 (11), pp. 533-547.

6. Lamm, R. (1989), "Public policy for an aging America», The Generational Joumal, 11 (1), pp. 108-111.

7. Preston, S.H. (1984), "Children and the eldetly in the US,, Scientific American, $250(6)$, pp. 44-49.

8. Callahan, D. (1987), Seting limits: medical goals for an aging society, NY, Simon and Schuster.

9. Nydegger, C.N. (1985), "Family ties of the aged in cross-cu tural perspective", en Hess, B.B. y Markson, E.W. (eds.), Growing old in Americt (3rd. edition), New Brunswick N.J, Transactior. Books.

10. Lopaca, H.Z. (1973), Widowhood in a US city, Cambridze MA, Schenkman.

11. Hess, B.B. y Markson, E.W. (1980), Aging and old age, N.w York, Macmillan.

12. Collins, R. (1985), Mariage and the Family, Chicago.

13. Bengston, V.L. y Dowd, J.J. (1980-1981), "Sociological funtionalism, exchange theory, and life-cycle analysis: A call for more explicit theoretical bridges", International Journal of Aging and Human Development, 12, pp. 55-73.

14. Cheal, D.J. (1983), "Intergenerational family transfers", Jo urnal of Marriage and the Family, 45, pp. 805-814.

15. Stoller, F.P. (1985), «Exchange patterns in the informal suppport networks of the elderly: The impact of reciprocity on moralem, Journal of Marriage and the Family, 47, pp. 335-342.

16. Hagestadt, G. (1987), «Parent-child relations in later life: Tsends and gaps in past research", en Lancaster, J.B., Altman, J.C., Rossi, A.C. y Shirrod, L.R. (eds.), Pa- 
"Papers»: Revista de Sociologia

renting across the life span: Biosocial Dimensions, pp. 405-434, New York, Aldine De Gruyter.

17. US Senate, Select Subcommirtee on Aging (1987), Developments in aging, Washington DC, US Government Printing Office.

18. Seccombe, K. (1988), "Financial assistence from ederly retirement-age sons to their aging parents", Research on Aging, 10 (1), pp. 102-118.

19. Stoller, E. y Pugliesi, K.L. (1988), "Informal networks of community-based ederly: changes in composition over timen, Research on Aging, 10 (4), pp. 499516.

20. Dewit, D.J. et. al. (1988), "Physical distance and social contact between elders and their adult children", Research on Aging, 10 (1), pp. 56-80.

21. Rosenmayr, L. y Kockeis, E. (1962), "Family relations and social contacts of he aged in Vienna", en Tibbets, C. y Donahuc, W. (eds.), Social and psychological aspects of aging, NY, Columbia University Press.

22. Pampel, F.C. (1981), Social change and the aged, Lexington MA, Lexington Books.

23. Hing, E. (1987), Use of nursing homes by the elderly: preliminary data from the 1985 National Nursing Home survey, National Center for Health Stadistics.

24. Worobey, J.L. y Angel, R. (1990), "Functional capacity and living arrangements of unmarried elderly persons", Journal fo Gerontology, 45, pp. 95-101.

25. Hcss, B.B. y Waring, J. (1984), "Family relationships of older women: a women's issue", en Markson, E.W. (ed.), Older women, Lexington MA, Lexington Books.

26. Stallones, L., Marx, M.B. y Garrity, T.F. (1990), "Prevalence and correlates of depresive symptorns among older US adults", American Journal of Preventive Medicine, 6(5), pp. 295-303.

27. Markides, K.S. y Levin, J.S. (1987), "The changing economy and the future of the minority aged", The Gerontologist, 27 (3), pp. 273-274.

28. Yee, B.W.K. (1990), "Gender and family issues in minority groups", Generations, XIV (3), pp. 39-42.

29. US Senate, Select Subcommittee on Aging (1988), Developments in aging, Washington DC, US Government Printing Office.

30. Worobey, J.L. y Angel, R. (1990), "Poverty and health: Older minority women and the rise of the female-headed household", Journal of Health and Social Behavior, 31, pp. 370-383.

31. Kasper, J.D. (1988), Aging alone: Profiles and projections, Baltimore MD, A report of the Commonwealth Found Commission on Fiderly P'cople Living Alone.

32. Antonucci, T. y Akiyama, H. (1987), "Social networks in adult life and a preliminary examination of the convoy model», Journal of Gerontology, 42, pp. $519-$ 527.

33. Braito, R. y Anderson, D. (1984), "The ever-single elderly women", en Markson, E.W. (ed.), Older women, Lexington MA, Lexington Books.

34. Stull, D.E. y Scarisbrick-Hauset, A. (1989), "Never-married elderly: a reassessment with implications for long-term care policy", Research on Aging, 11 (1), pp. i24-139. 
35. Wolf, J, et al. (1983), «Distance and contacts: Interactions of Black urban elderly Adults with family and friends", Joumal of Gerontology, 38 (4), pp. 465-471.

36. Shaw, L.B. (1986), Midlife women at work: A fifteen-year perspective, Lexington MA, DC Health and Company.

37. Report of the Secretary of Labor (1989), Labor market protlems of older workers, Washington DC, US Dept. of Labor.

38. National Research Council (1988), The aging population in the twenty-first century: Stadistics for health policy, Washington DC, National Acadamy Press.

39. Treas, J. (1977), "Family support systems for the aged: Some social and demographic considerations", Gerontologist, $17(6)$, pp. 486-491.

40. Lopata, H.7. (1990), "Which child: the consequences of sucial development on the support system of widows", en Hess, B.B. y Markson, 'E.W. (eds.), Growing old in America (4th edition), New Brunswich, NJ and Lonkion, Transaction Publishers.

41. Kivett, V. (1985), "Consanguinity and kin level: they relative importance to the helping network of older adults", Joumal of Gerontology, 40 (2), pp. 228-234.

42. Soldo, B.J. y Manton, K.G. (1985), "Health status and service needs of the oldestold: current patterns and fucure trends", Milbank Memorial Fund Quarterly, 63.

43. Qureshi, H. y Waller, A. (1987), The caring relationship, London, Routledge and Kegan Paul.

44. Cicirelli, V.G. (1983), "A compartsion of helping behavios to elderly parents of adult children with intact and disrupted marriages", Gerontologist, 23, pp. 619-625.

45. Ryan, R.; Hooyman, N. y Briar, K.H. (1985), "Women as caregivers of the elderly: the historical legacy of private burden", Paper presented at the CSWE Symposium on Women's issues.

46. Ikels, C. (1985), "The process of caretaker selection", en Fess, B.B. y Markson, E.W. (eds.), Growing old in America (3rd edition), New Brunswich NJ, Transaction Publishers.

47. Fengler, A.P. y Goodrich, N. (1979), "Wives of elderly disabled men: The hidden patients", The Gerontologist, 20, pp. 649-655.

48. Zarit, S.H.; Reeves, K.E. y Bach-Peterson, J. (1980), "Relitives of the impaired elderly: Correlates of feelings of burden", The Gerontologist, 20, pp. 649-655.

49. Cantor, M.H. (1983), "Strain among caregivers: A study of txperience in the Un:ted States", The Gerontologist, 23, pp. 81-93.

50. Zarit, S.H. (1989), "Do we need another "stress and caregiving" study?", The Gerontologist, 29 , pp. 147.

51. Pearlin, L.I.; Mullan, J.T.; Semple, S.J. y Skaff, M.M. (1990), "Caregiving and the stress process: An overview of concepts and their measuress, The Gerontologist, 30, pp. 583-594.

52. Horowirz, A. (1985), "Sons and daughters as caregiving to older parents: differences in role performance and consequences", Gerontoloçist, 25, pp. 612-617.

53. Brody, E. et al. (1983), "Women's changing roles and help to elderly parents: attitudes of three generations of women", Journal of Gerontslogy, 38 (5), pp. 597 607. 
"Papersn: Revista de Sociologia

54. Schoonover, C.B. et al. (1988), On borrowed time: How the growth in entitlement spending threatens America's future, San Francisco, ICS Press.

55. Chodorew, N.J. (1978), The reproduction of mothering, Berkeley, University of California Press.

56. Population Research Bureau (1990), World population data sheet 1990, Washington DC Population Rescarch Bureau.

57. Robinson, B. y Thurnher, M. (1979), "Taking care of parents: a family cycle transition", Gerontologist, 19, pp. 586-593.

58. Statistical Abstract of the United States 1990(1990), Washington DC, US Government Printing Office.

59. Sidel, R. (1986), Women and children last: The plight of poor women in affluent America, New York and London, Penguin Books.

60. US Joint Economic Committee, US Congress (1986), The Conception of Wealth in the United States, Washington DC, Joinc Economic Commitree.

61. Avery, R.B. y Elliehausen, G.E. (1989), "Financial characteristics of high income families", Federal Reserve Bulletin (march), pp. 163-176.

62. Avery, R.B. y Kennickel, A.B. (1989), "Rich rewards", American Demographics (june), pp. 19-22.

63. US House of Representatives, Committee on Ways and Means (1989), Background material and data on programs within the jurisdiction of the Committee on Ways and Means, Washingron DC, US Goverment Printing Office. 